\title{
PUESTA EN VALOR Y USO SOCIAL DEL SISTEMA DE ANDENERÍAS AGRÍCOLAS DE LA ZONA ARQUEOLÓGICA DE URCO, SECTOR II QOSQOPATA, CALCA ${ }^{(*)}$
}

\author{
VALUE ENHANCEMENT AND SOCIAL USE OF THE AGRICULTURAL TERRACE IRRIGATION \\ SYSTEM OF THE ARCHAEOLOGICAL SITE OF URCO, SECTOR II QOSQOPATA, CALCA \\ SHELBY PAZ VALENCIA ${ }^{(* x)}$
}

Fecha de recepción: 11 de mayo de 2016

Fecha de aprobación: 22 de junio de 2016

\section{RESUMEN}

La conservación del patrimonio sería un acto incompleto si no estuviese acompañado de actividades sociales y ambientales en cada lugar. La apertura de los bienes patrimoniales es el primer paso; su aprehensión, el segundo, es decir, su 'entendimiento'. La presente propuesta persigue la aproximación al planteamiento y desarrollo de un proyecto de puesta en valor que, desde un enfoque multidisciplinario, permita el uso social y la revaloración de un recurso arqueológico de forma que se reconozca y fortalezca los valores endógenos de la comunidad que lo alberga. Además, se pretende que este proyecto logre la rehabilitación de un sistema de andenerías mediante principios restaurativos que generen dinámicas económicas que puedan interactuar con una dialogante oferta turística responsable que proteja y mantenga los sitios arqueológicos, para así insertar a su comunidad en los trabajos de puesta en valor, y su posterior uso y aprovechamiento. La principal finalidad del proyecto es constituirse como uno de los testimonios agrícolas más importantes del circuito del valle del Vilcanota.

\section{PALABRAS CLAVE}

Puesta en valor, uso social del patrimonio, andenes agrícolas

\section{ABSTRACT}

Heritage conservation would be an incomplete act if it were not accompanied by the social and environmental activities of each place. The opening of the assets is the first step; its apprehension the second (that is, its "understanding"). This proposal chases after the proposal and development of a value enhancement project, which -after a multidisciplinary approach- allows the social use and value enhancement of an archaeological asset. This must be done in a way that recognizes and strengthens the endogenous values of the community that houses the project. As well, this project aims to achieve the rehabilitation of a system of irrigation terraces through restoration guidelines that generate dynamic economic principles. In turn, these can interact with a responsible tourism that generates dialogue, and protects and maintains. This way it can insert its community in value enhancement projects, and their use and revaluation. The main purpose of the project is to establish itself as one of the most important agricultural records of the Vilcanota Valley circuit.

\section{KEYWORDS}

Value enhancement, social use of heritage, agricultural terraces

(*) El presente artículo fue desarrollado sobre la base del proceso de puesta en valor de una zona arqueológica en el valle del Vilcanota (2009-2012), como parte de los proyectos desarrollados por el PER Plan Copesco-Cusco, en el marco del préstamo del BIF y el Estado peruano, proyecto que vinculó la revaloración cultural y la promoción turística promoviendo la participación de la comunidad integrada a su patrimonio como principal actor. (**) Arquitecta titulada por la Universidad Nacional San Antonio Abad del Cusco, egresada de la Maestría de Gestión Cultural de la Universidad Nacional Mayor de San Marcos. Residente de diversos proyectos de conservación, restauración de sitios arqueológicos como Choquequirao y el valle de Vilcanota. Becaria participante de cursos de especialización en restauración y conservación de patrimonio desarrollados en el Instituto Nacional de Antropología e Historia (INAH) - México Latam, y en el Centro Internacional de Estudios para la Conservación y la Restauración de los Bienes Culturales (ICCROM) Roma. Actualmente labora en el Ministerio de Cultura del Perú. Contacto: chelbichita@gmail.com 
La zona arqueológica de Urco es un complejo sistema de andenerías y terrazas prehispánicas que fueron construidas con fines agrícolas, para el manejo de los diversos sistemas de regadío y cultivo, que gracias al manejo por parte de la comunidad que allí reside conserva gran parte de su estructura actualmente. Sin embargo, pese a ser una de las más grandes infraestructuras agrícolas ubicadas en el camino Calca - Urubamba, ha sido muy afectada por el tiempo y los procesos de urbanización que han tenido lugar en la provincia de Calca.

Su cercanía al complejo de Huchuyqosqo, ubicado a $3 \mathrm{~km}$ de distancia, permite asociar su tipología constructiva y de manejo de tierras con el territorio que dominaron los incas, sumándose así a parte de los hitos arqueológicos atribuidos a tal cultura que se encuentran ubicados en el valle del Vilcanota.

El valle del Vilcanota, con el paso del tiempo, ha sufrido diversos impactos, como su desconfiguración territorial; el acelerado y descontrolado crecimiento de sus poblaciones transformó y reconfiguró el espacio agrícola por sectores de vivienda. Por otra parte sitios arqueológicos como Pisac, Ollantaytambo y Huchuyqosqo, ubicados en este segmento territorial, generaron una oferta turística y una dinámica económica creciente puntual que logró excluir rápidamente a las poblaciones cercanas ( $\mathrm{Paz} \mathrm{Va}$ lencia \& Samaniego Mollinedo, 2011).

Este panorama exige programas de desarrollo y manejo integral que involucren tanto el planteamiento urbano como el manejo de las zonas arqueológicas y sus comunidades beneficiarias. De forma complementaría se exige una búsqueda por incluir la protección y conservación de sus diversos valores arqueológicos, históricos y ecológicos, de manera que se constituyan en la columna principal del cambio social y el mejoramiento de la calidad de vida de la población, resaltando su potencial arqueológico, histórico, turístico y social.

En este contexto el Proyecto Vilcanota, proyecto especial del PER Plan COPESCO', como respuesta a esta necesidad plantea el Proyecto de Reordenamiento y Rehabilitación del Valle del Vilcanota, en el cual se elaboran dos proyectos pilotos que tienen por objeto fomentar iniciativas de desarrollo sostenible en este valle. Reconociendo los valores y potencialidades de este sector, se considera que incluye en su estructura los componentes sociales y ambientales, estudio de medios y soportes que aporten a su uso responsable, a través de la promoción de la preservación cultural como eje primigenio. El desarrollo turístico, la infraestructura urbana, la protección ambiental y el desarrollo social son las sinergias motoras que enriquecen y complementan este proyecto.

Luego del primer acercamiento y reconocimiento al sitio, se promueve la elaboración del expediente técnico denominado "Restauración y Puesta en Valor de la Zona Arqueológica Monumental Urco Sector I Qosqopata, Calca". Para plantear y acompañar el desarrollo de las diversas actividades y trabajos que conformaron este ambicioso proyecto se convocó un equipo multidisciplinario conformado por arquitectos, arqueólogos, ingeniero civil, ingeniero geólogo, biólogos, y antropólogos, con el objetivo de potenciar el valor arquitectónico y arqueológico del sitio, su sistema de andenerías y plataformas de cultivo; promover su actual uso agrícola; y resaltar su significancia y connotación cultural en el valle. Todo ello con el fin de que se constituya como uno de los testimonios agrícolas más importantes del circuito del Valle del Vilcanota.

1. Es una entidad especializada del Gobierno Regional Cusco, ha sido la entidad del estado encargada de sentar las bases para el desarrollo turístico de la región, a través de la restauración y puesta en valor de monumentos, así como de planificación, estudio y construcción de infraestructura turística, en acciones concertadas con otras entidades del Estado y de la sociedad civil involucradas en la gestión del turismo. 


\section{Ubicación y contexto del proyecto}

En la ciudad del Cusco, hacia el noreste fuera de la ciudad, se encuentra el Valle del Vilcanota, el área que se ubica hacia las riberas del río que lleva el mismo nombre. Se trata de un valle interandino que se caracteriza por su geología, así como por los depósitos y conos aluviales cuaternarios que forman sus riberas. Estas formaciones albergan el complejo arqueológico denominado Urco, localizado en el distrito de homónimo, en la provincia de Calca. Se trata de un sistema de andenerías construido en las riberas del río Vilcanota, a una altura de 2993.50 m.s.n.m., conformado por plataformas destinadas al aprovechamiento agrícola (ver Figura 1).

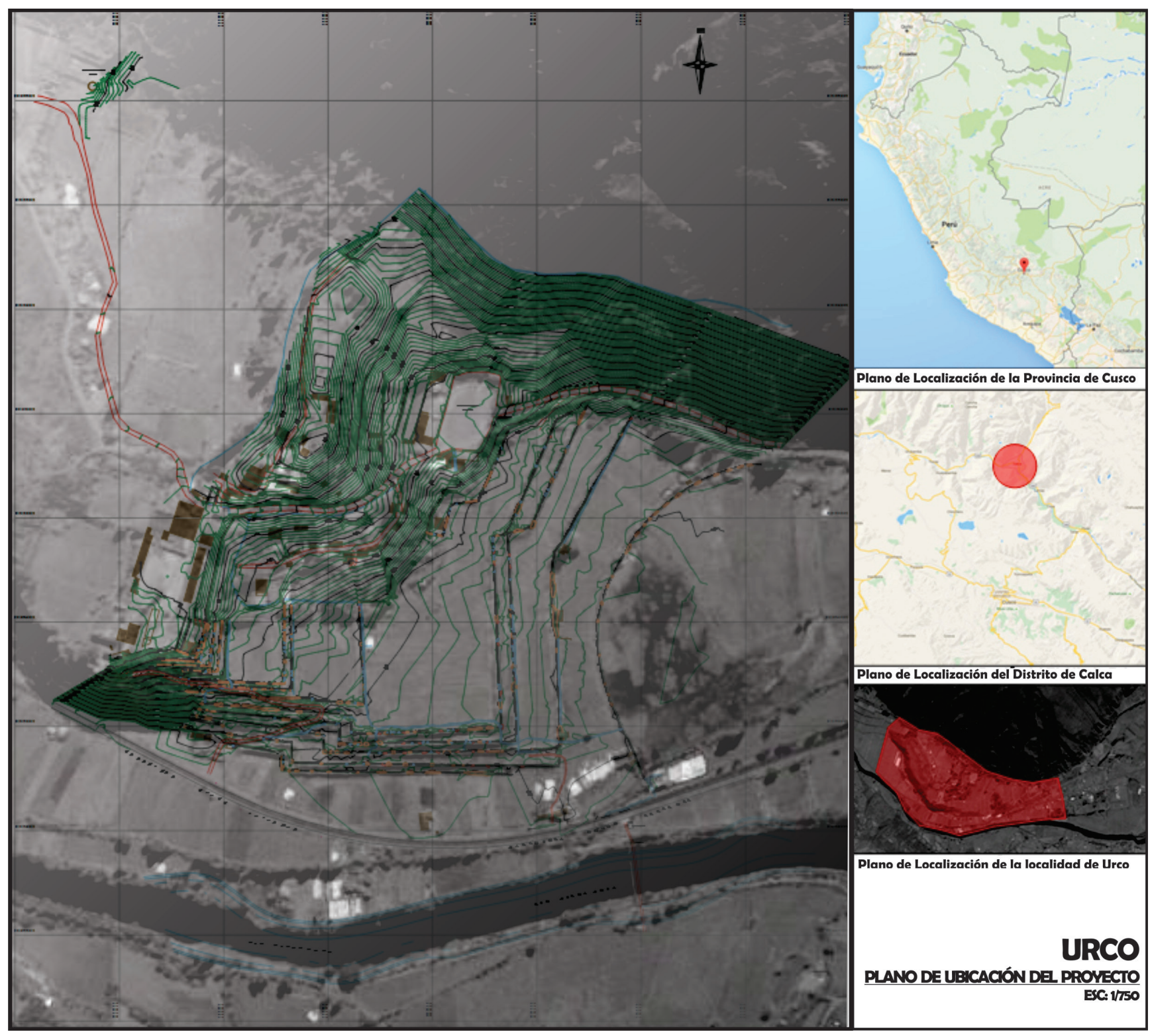

Figura 1. Localización del complejo arqueológico

Fuente: Archivo institucional PER Plan COPESCO, 2010. 
devenir Vol. 3, N5, ENERO - JUNIO 2016, PP. 133-154 - EstudIOS | ISSN 2312-7570

UNIVERSIDAD NACIONAL DE INGENIERÍ, LIMA

\section{Contexto histórico}

El complejo arqueológico de Qosqopata refiere su filiación cronológica a la época inca, debido a las referencias de su uso y construcción encontradas en diversas crónicas al inca soberano Urco Huaranca, segundo hijo del Inca Huiracocha, el cual eligió el sector de Calca para asentar su linaje en honor al apu Pitusiray, alrededor del cual se originan diversos mitos (Víctor, 1988).

Los apus Pitusiray y Sawasiray, situados en Calca, fueron considerados según Guamán Poma como ídolos y guacas importantes dentro de la ideología inca, ya que se menciona que Guayna Cápac, onceavo gobernante, el menor de ellos, fue quien se encargó de consolidar la región inca en estos pueblos: "...relojes agrícolas creados a comienzos del siglo XV por el noveno inca Pachacuteq hijo del Inca wiracocha y hermano de urco Huaranca, relojes llamados pacha unanchaq, hechos de cantería inca sobre los cerros más altos de las cumbres" (Betanzos, 1987, p. 10).

Durante el periodo de la Colonia, este sector fue explotado y aprovechado debido a su gran productividad agrícola, por lo que se constituyó en un sector importante para la época y el sector de Calca. Este factor fue determinante para las reformas dadas posteriormente durante el Gobierno de Velasco Alvarado, cuando se promulgó la Ley de Reforma Agraria, en 1969, cuyo objetivo principal era reorientar los recursos capitales hacia un régimen justo de tenencia de tierra para los más pobres. Así, estos territorios fueron divididos y distribuidos entre diversas asociaciones de trabajadores dedicados a la producción agropecuaria que, finalmente, en muchos casos no fueron exitosos (ver Figura 2).

El manejo y aprovechamiento de este sistema de andenerías actualmente se encuentra a cargo de la cooperativa denominada 'Cristo Salvador de Urco', la misma que año tras año en el mes de octubre evoca el mito de 'Unu Urco', festival de culto al agua que tuvo por objeto la apertura de canales para el tránsito del agua desde su fuente principal, ubicada en la laguna Pawaray (ver Figura 3).

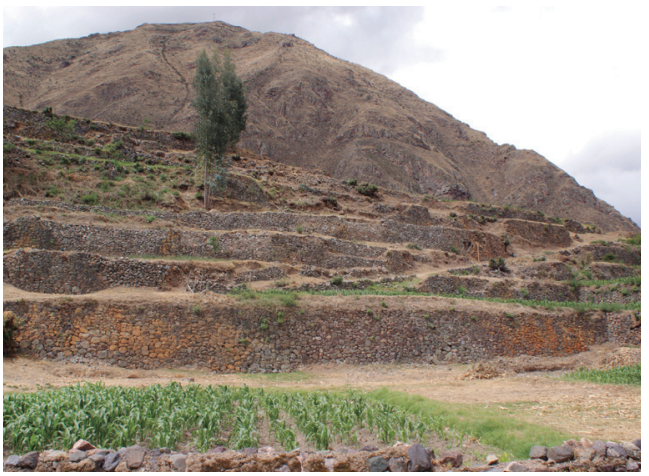

Figura 2. Estado de los andenes antes de la intervención. Vista de tramos alterados, apertura y corte para accesos vehiculares de plataformas de cultivo Fuente: Archivo fotográfico Shelby Paz, 2010.

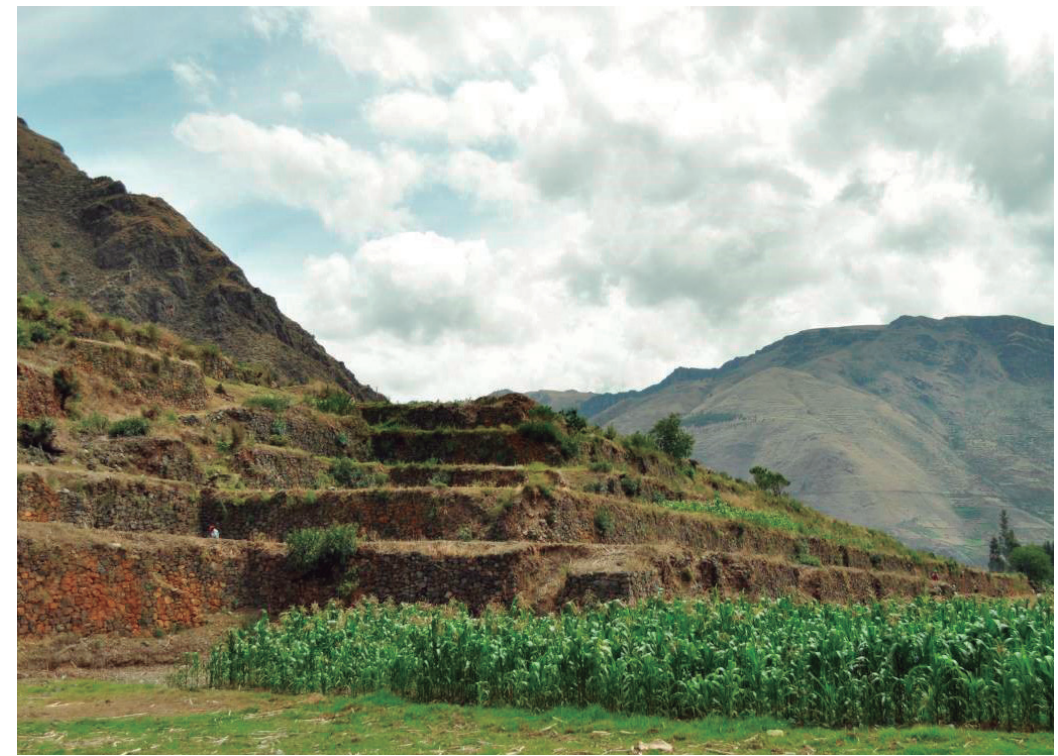

Figura 3. Estado de los andenes antes de la intervención. Vista de las plataformas sin uso, hacia la base cultivos de maíz

Fuente: Archivo fotográfico Shelby Paz, 2010. 

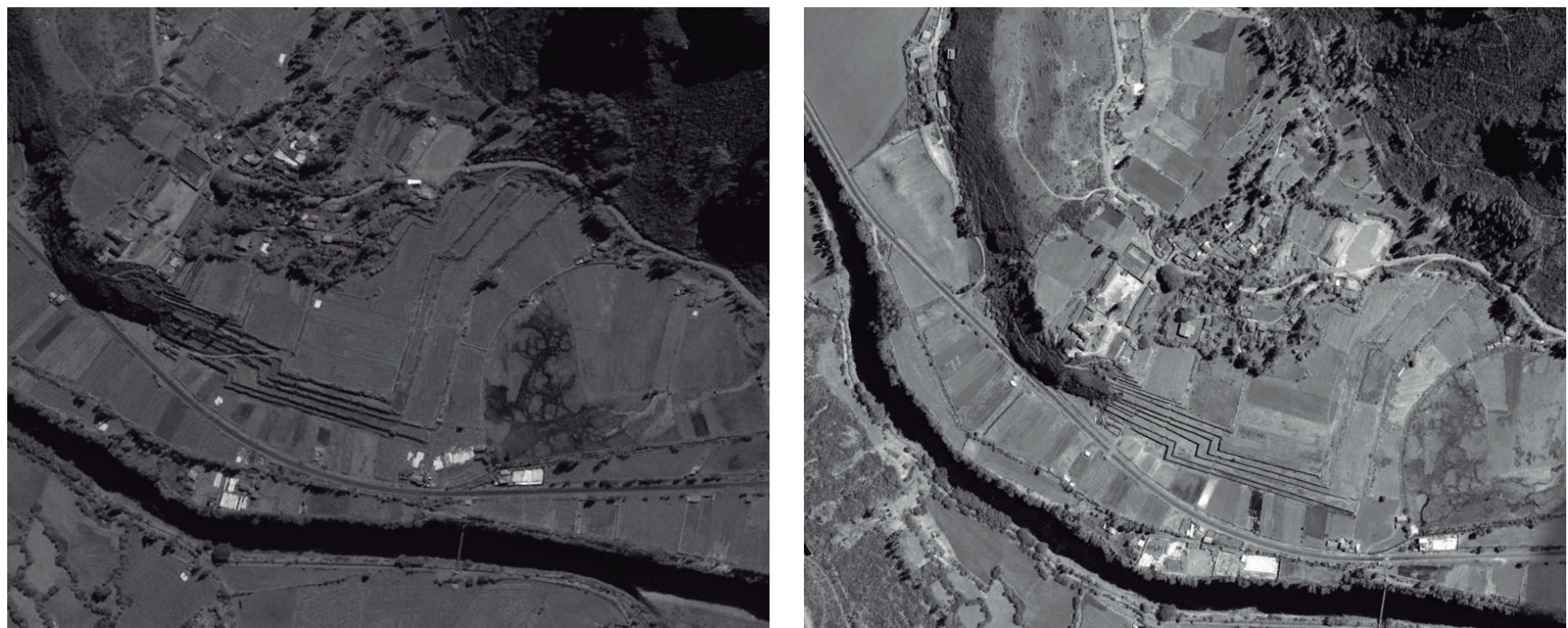

\section{Contexto urbano y emplazamiento}

"Los territorios conquistados por los incas a lo largo del valle del Vilcanota, exigió el emplazamiento de establecimientos y centros urbanos, que lograron su sostenibilidad durante el imperio, muchos de ellos perduraron en la colonia..." (Canziani, 2009, p. 448).

La inserción de ganadería posterior a la Colonia activó la explotación agrícola y el manejo desmedido de las tierras agrícolas, lo que generó un fuerte impacto en la zona arqueológica, la cual había mantenido intacto gran parte de su territorio hasta inicios del siglo XVIII.

A inicios del siglo XIX, la zona arqueológica se vio afectada por el abandono y la expansión urbana, la cual se dio de forma desmedida; el cambio de uso de suelo y las leyes de favorecimiento de tenencia de tierras aceleraron diversos factores de deterioro en el sistema de andenerías.

En el año 1990, la inserción de maquinaria pesada (tractores), que facilitó y aceleró los prácticas agrícolas de siembra y cosecha, generó expectativa en cuanto al mejor aprovechamiento de las tierras. Sin embargo, el resultado fue totalmente contrario, pues se afectó directamente las estructuras de los andenes como consecuencia de la apertura de caminos y tramos vehiculares, que desfiguraron el planteamiento original y empobrecieron la tierra.

Actualmente, la falta de planes urbanos en los diversos centros urbanos del valle del Vilcanota ha provocado una expansión urbana desordenada. Asimismo, el cambio de usos de suelo ha ocasionado la alteración de sectores con valor arqueológico, sobre todo en el caso de Urco. Estos factores han determinado la pérdida de grandes sectores agrícolas, como ocurre hacia la parte superior de los andenes, donde se ha establecido una configuración urbana que responde al asentamiento contemporáneo de la zona (ver Figuras 4 y 5 ).

\section{Descripción arquitectónica de la Zona Arqueológica de Urco - Qosqopata}

La configuración del complejo arqueológico de Urco presenta dos importantes sectores y patrones de asentamiento inca correspondientes al período tardío, los cuales se logran identificar por su configuración arquitectónica y urbana (Gasparini, 1997).
Figura 4. Vista del estado actual de la lectura formal y urbanización (fotografía satelital, 2008)

Fuente: Portal Terra Server https://www. terraserver.com/view?utf8=\%E2\%9C\%93\&search_text=calca\%2C+cusco\%2C+peru\&searchLat $=\&$ search $L n-$ $\mathrm{g}=\&$ lat=\&lng=\&bbox=\&center=, 2016 .

Figura 5. Recuperación de las plataformas, y la lectura de emplazamiento (fotografía satelital, 2012)

Fuente: Portal Terra Server https://www. terraserver.com/view?utf8=\%E2\%9C\%93\&search_text=calca\%2C+cusco\%2C+peru\&searchLat $=\&$ search $L n-$ $\mathrm{g}=\& l a t=\& \operatorname{lng}=\& b b o x=\&$ center=, 2016 . 

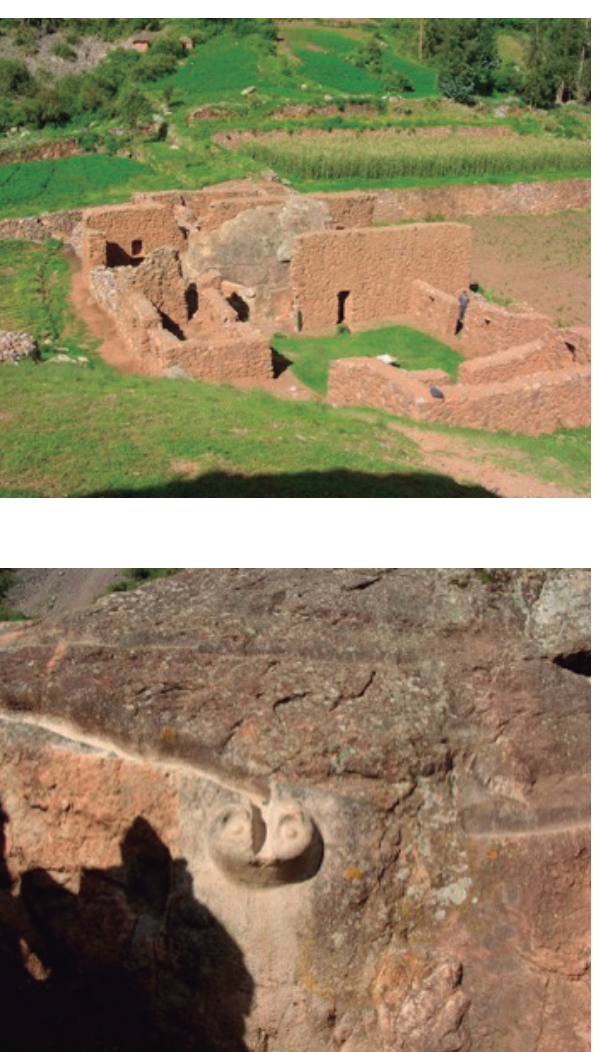

Figura 6. Recintos ubicados hacia el noreste del complejo arqueológico Fuente: Archivo Institucional PER Plan Copesco, 2010.

Figura 7. Pieza lítica con representación zoomorfa

Fuente: Archivo Institucional PER Plan Copesco, 2010.
1. Sector I, sector ceremonial. Ubicado hacia el noreste del sitio arqueológico delimitado como Urco, en él se encuentran diferentes recintos construidos en adobe y piedra. Su diseño y emplazamiento ortogonal guardan correspondencia con la simetría y organización de las canchas incas de la ciudad del Cusco (Gasparini, 1997). Este emplazamiento se complementa con espacios y ambientes de diseños circulares, vinculados con adoratorios y almacenes de granos. Alrededor de este conjunto edilicio se encuentra un monolito tallado en piedra que representa un ser zoomorfo que está asociado al mito de Unu Urco (ver Figuras 6 y 7 ).

2. Sector II, sector agrícola. Ubicado hacia el sureste del sitio arqueológico, se caracteriza por el aprovechamiento de un área coluvial (Dorado Alegre, 2010), sobre la cual se construyó un sistema de plataformas y andenes agrícolas ubicado hacia el Este en la base del apu Pitusiray, con un entramado de canales de agua para regadío que desciende desde la laguna de 'Kan Kan'.

Los muros de contención que soportan el sistema de andenerías están constituidos por líticos de tipo diorítico, asentados de forma rústica. En conjunto, estas plataformas poseen una distribución escalonada que simula una pirámide trunca. Las diferentes referencias históricas realizadas por cronistas evidencian que las tradiciones aún se mantienen vivas en la comunidad, la cual las hace aún parte viva de sus ciclos culturales más importantes, como es la festividad del 'Unu Urco' (ver Figura 8).

La conformación estructural de este sistema de andenerías resalta el trabajo realizado para su construcción, que se basa en un sistema de recirculación y control de aguas subterráneas, mediante el manejo de diferentes estratos y rellenos artificiales de terreno que cumplieron eficazmente sus funciones agrícolas.

Las características de su construcción dieron forma al uso de muros de contención levemente inclinados, que a su vez forman los taludes y superficies horizontales de tratamiento agrícola, lo que permite la distribución del riego y el manejo eficaz del terreno. Asimismo, mediante un paramento rústico semicircular que bordea un humedal abastecido de ocho manantiales subterráneos, ubicado hacia la base de las plataformas, se aseguró el abastecimiento y circulación del agua de forma aleatoria para las grandes plataformas.

De acuerdo a las características constituyentes de los andenes identificadas en este sistema se determina, de acuerdo a la clasificación tipológica de Kendall, que estos corresponden al "tipo 1" (Kendall \& Rodríguez, 2009). La conformación de esta tipología se da en tres niveles de relleno artificial de forma ascendente. Se inicia con una nivelación horizontal de una capa de grava gruesa, otra capa de limo arenoso y finalmente la capa de la tierra humosa. Esta tecnología llega a combinarse con el uso de canales principales y secundarios, que abastecen de forma continua las plataformas en sus diferentes niveles (Venero Oblitas, 2010). Las excavaciones arqueológicas correspondientes al Proyecto de Investigación Arqueológica permitieron la observación constante de estas características, lo cual significó un aporte importante durante el proceso de intervención. Dicho proceso también acompañó el trabajo de puesta en valor, y permitió rescatar la tipología estratigráfica para la conformación de las plataformas (ver Figura 9).

El diseño de los andenes y los elementos complementarios corresponden a un estilo arquitectónico imperial inca, de uso ideal para la construcción de los mismos en laderas escalonadas. En este caso específico, la construcción es de mucha importancia, ya que los andenes presentan un trabajo particular en el sistema de circulación e interconexión vertical, como las escalinatas embutidas en muro y las sarunas, así como en el manejo de los sistemas de riego vertical (Venero Oblitas \& Samaniego Mollinedo, 2010).

Funcionalmente, este sistema de andenería combinó elementos arquitectónicos secundarios, como los elementos de accesibilidad vertical, constituidos por escalinatas de piedra embutidas en los paramentos y sarunas, distribuidas diagonalmente en 


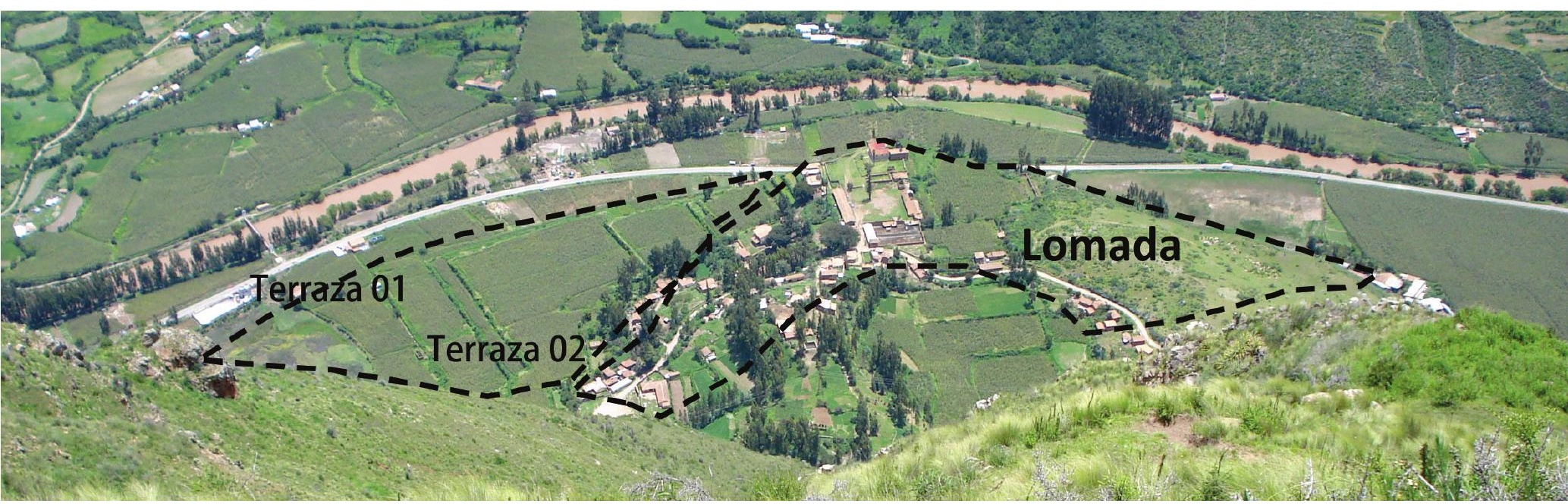

los muros de los diferentes niveles de las plataformas. Ambos componentes se encuentran repartidos simétricamente a lo largo de los muros, y combinan su posición con canales verticales que distribuyen y organizan el recorrido del agua que proviene desde lo alto (ver Figura 10).

\section{Descripción de la intervención en el sector II, Sistema de andenerías}

El proyecto propuso la rehabilitación y recuperación de las plataformas de cultivo, que incluyó, además, la recuperación del sistema hidráulico original y, de forma complementaría, la implementación de un sistema de riego alterno. Se consideró también la recuperación de los sistemas de circulación vertical (escalinatas embutidas y sarunas) y de circulación horizontal (implementación de sendas peatonales). Todo ello se llevó a cabo bajo rigurosas acciones de investigación arqueológica y arquitectónica de las estructuras soterradas (Paz Valencia \& Samaniego Mollinedo, 2011).

Como parte del soporte técnico, se implementaron componentes sociales y medioambientales que complementaran las acciones contempladas para los fines de restauración y puesta en valor de la zona arqueológica de Urco, lo cual exigió el establecimiento de un equipo multidisciplinario.

La recuperación tipológica del esquema espacial y funcional original del sistema de andenes, así como el fortalecimiento de capacidades de la comunidad, pretendía implementar un modelo de intervención que debía respetar y rescatar la actividad agrícola dentro del contexto del valle Vilcanota. Así también, se enfatizó su valor en cuanto autenticidad, mediante la eliminación de los sectores precarios de vivienda que se ubicaban en algunos sectores del sistema de andenes, y la recuperación de su conformación arquitectónica.

El mejoramiento del riego y aprovechamiento racional de los recursos, a través de la implementación de técnicas y tecnologías de cultivo que armonicen el uso agrícola de la tierra y la conservación del sector arqueológico, permitirían su mantenimiento y conservación. Esto, además, potenciaría un nuevo producto turístico responsable, que combine los diversos aspectos culturales y genere una actividad económica sostenible para la comunidad, la que contribuiría a la salvaguarda y revaloración de su patrimonio inmaterial.

El área propuesta para la intervención dentro del sitio arqueológico correspondió al sector agrícola, con una extensión de 17,135 $\mathrm{m}^{2}$ aproximadamente, distribuida en 11 niveles y con una longitud que sobrepasa los 200 metros lineales en cada plataforma (Venero Oblitas \& Samaniego Mollinedo, 2010).
Figura 8. Vista general desde el noreste de las plataformas, se describe la formación aluvial del asentamiento Fuente: Archivo Institucional Proyecto Vilcanota, 2010

Figura 9. Conformación estratigráfica Fuente: Archivo fotográfico Shelby Paz, 2010.




devenir Vol. 3, N5, ENERO - JUNIO 2016, PP. 133-154 - ESTUDIOS | ISSN 2312-7570

UNIVERSIDAD NACIONAL DE INGENIERÍ, LIMA

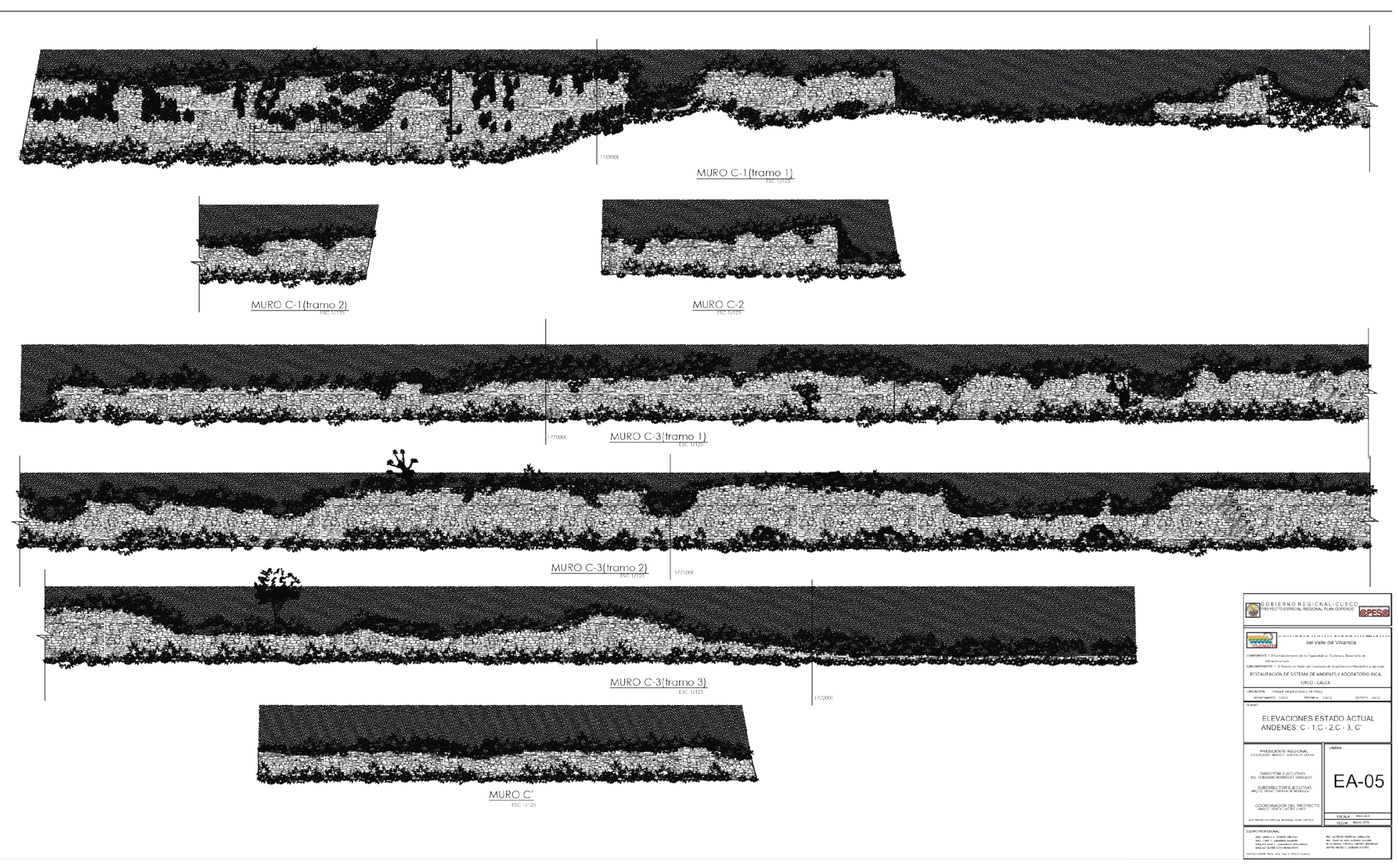

Figura 10. Lámina de relevamiento del estado actual de muros de andenes

Fuente: Archivo institucional PER Plan Copesco, 2011.

\section{Metodología de intervención}

Las acciones contempladas por el proyecto se dividieron en dos ejes principales, como resultado de la identificación de los principales factores de deterioro a nivel general en el área propuesta. El primer eje fue el componente de intervención técnica, planteada mediante una metodología sistemática cuya importancia radicó en la correcta documentación, registro arquitectónico y patológico de cada elemento. Fue desarrollado de la siguiente manera:

- Identificación de tramos y lesiones

- Codificación y reticulado, tramos de 2 a 5 metros

- Dibujo a mano alzada

- Registro fotográfico de cada tramo

- Corrección fotográfica

- Colocación de balizas (guías de madera), trazado de pendientes e inclinación de muros

- Trazado de niveles de piso y coronamientos de muro

- Definición de intervenciones por anastilósis como consolidación, restauración, reintegración, restructuración estructural y restitución (ver Figuras 11, 12, 13, 14, 15 y 16)

El segundo eje de la intervención lo constituyeron el componente medioambiental y el componente social, los cuales aportaron en la recuperación de los valores endó- 


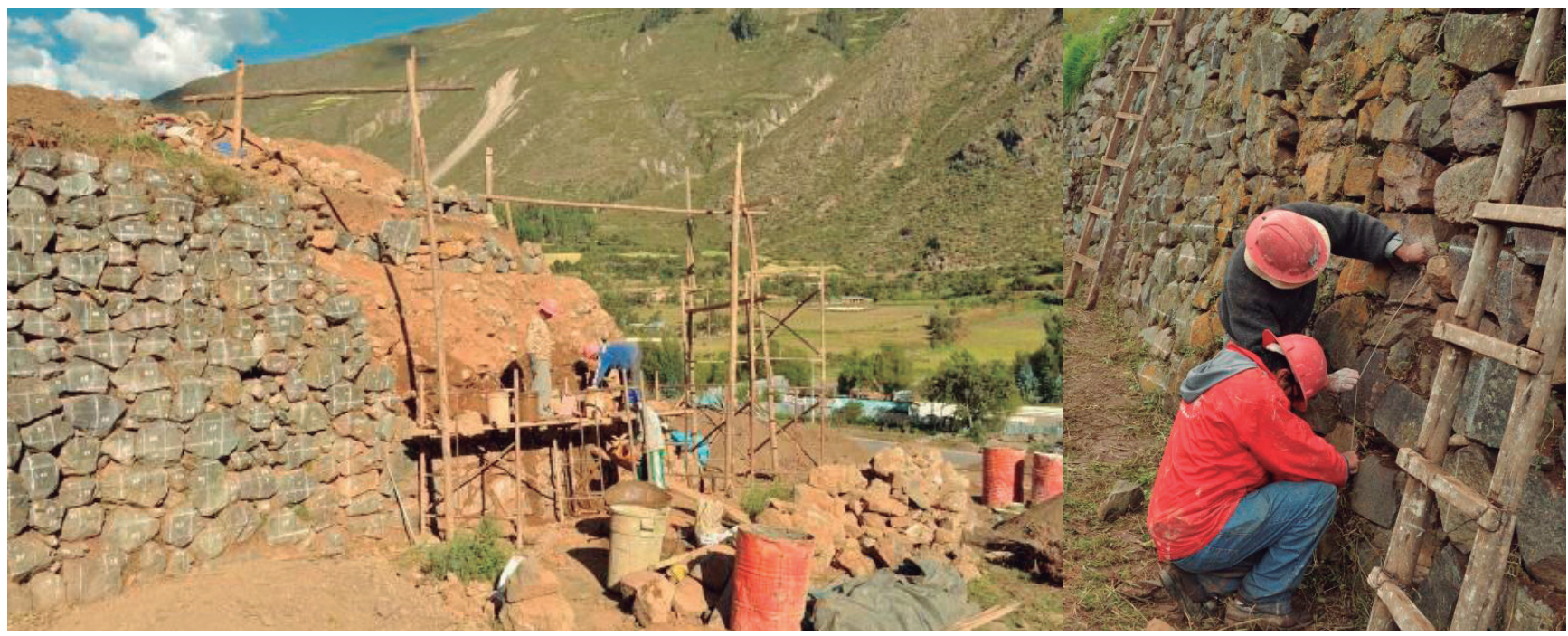

Figuras 11 y 12. Procesos de codificación y dibujo a mano alzada

Fuente: Archivo fotográfico Shelby Paz, 2010.



Figuras 13 y 14. Identificación de lesiones

Fuente: Archivo fotográfico Shelby Paz, 2010.

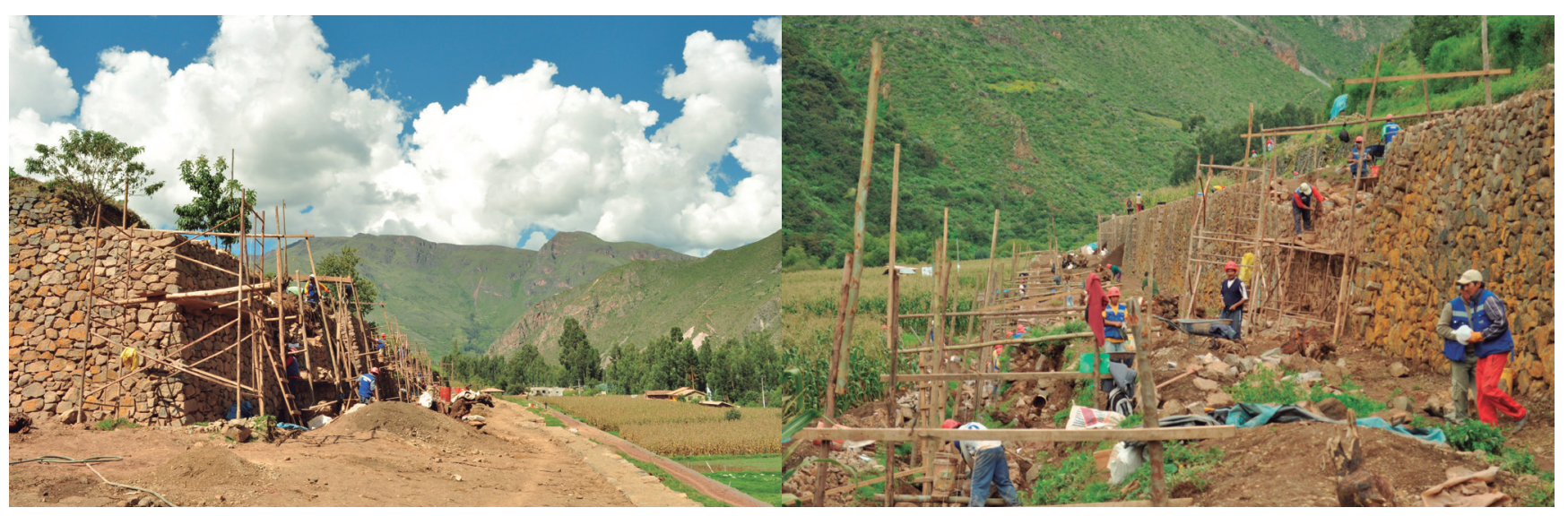

Figuras 15 y 16. Colocación de balizas, guías y andamios para iniciar el trabajo

Fuente: Archivo fotográfico Shelby Paz, 2010. 
genos de la comunidad mediante la implementación de talleres de concientización y trabajo comunal. Con esto se buscó incidir en la apropiación y reconocimiento de los valores del bien cultural por parte de la comunidad. Con este fin, se involucró a entidades estatales como actores estratégicos, tales como la Municipalidad de Calca, el Ministerio de Cultura e instituciones vinculadas al agro, como el Instituto Nacional de Innovación Agraria (INIA) (Paz Valencia \& Samaniego Mollinedo, 2011).

\section{Criterios de intervención}

Para la intervención adecuada del monumento, y como parte primordial del proyecto, resultó imprescindible desarrollar la evaluación del nivel y tipo de acciones a realizar, así como lograr armonizar los requerimientos metodológicos de la investigación científica con los principios teóricos de la restauración. Estos enfoques se desarrollaron dentro de un contexto en el que uno de los pilares fundamentales fue la recuperación de las principales prácticas comunales vinculadas a la agricultura, todo esto con un enfoque y discusión multidisciplinar. De este modo se plantearon algunos criterios de intervención:

- La restauración en los andenes contempla acciones dirigidas a devolver la integridad estructural y física óptima, ello siempre con un carácter multidisciplinario que busque conservar los valores arquitectónicos, estructurales, históricos y funcionales del monumento mediante intervenciones que logren devolver estas características a los mismos.

- Por conservación, en el contexto del proyecto, se entiende el producto de un conjunto de acciones que la comunidad realizará para lograr que su patrimonio y sus monumentos perduren (Petzet, 2000). Estas operaciones de conservación tienen como finalidad prolongar y mantener el mayor tiempo posible los materiales de los que están constituidos los andenes. Para lograr este objetivo, se plantea una estrategia de intervención compuesta por los siguientes factores: el análisis de los factores de deterioro, el control del estado de conservación, el control de las condiciones medioambientales y la intervención sobre el medioambiente.

- La rehabilitación de andenes está referida a reestablecer o recuperar la función agrícola original del andén. Es decir, utilizar esta infraestructura para los fines agrícolas para los que fue construida.

- El retiro o demolición de edificaciones que afecten la armonía y configuración espacial de los andenes. Se identifican estructuras de vivienda y cercos perimétricos adosados a los paramentos que deberán ser retirados.

- Facilitación de la circulación peatonal, que consiste en el establecimiento de circuitos y sendas de circulación para los comuneros en sus áreas de cultivo.

Además, según la Carta de Cracovia, se considera intervenciones a muchas de estas operaciones, tales como el control medioambiental, mantenimiento, reparación, restauración, renovación y rehabilitación. Sin embargo, el mantenimiento y reparación son parte sustancial del proceso de conservación, por lo que estas intervenciones deben fundamentarse en investigaciones arqueológicas sistemáticas, inspecciones, control, seguimiento y pruebas de materiales, todo esto con el objetivo de tomar las medidas correctivas durante el proceso de intervención y, a su vez, realizar las propuestas y estrategias de conservación a llevarse a cabo a largo plazo (Venero Oblitas \& Samaniego Mollinedo, 2010).

\section{Objetivos de la intervención}

El objetivo principal de la intervención es la puesta en valor del sistema de andenes agrícolas mediante acciones de restauración que, sobre la base de los criterios plantea- 
dos y un enfoque multidisciplinario, revaloren su significancia y uso mediante el mejoramiento de cosechas y sistemas alternativos de riego, y se promuevan así cultivos ecológicos y alternativas de turismo vivencial.

Estos trabajos de conservación se relacionan con las capacidades endógenas de los miembros de la comunidad, al integrarlos al grupo de trabajo de obra, en concordancia con la Carta Internacional para la Gestión del Patrimonio Arqueológico, propuesta por el Comité Internacional para la Gestión del Patrimonio Arqueológico (ICAHM) en Lausana en 1990. Esta indica que la participación activa de la población debe incluirse en las políticas de conservación del patrimonio arqueológico, pues generar el compromiso y participación de la población local es un medio efectivo para la promoción del mantenimiento de las edificaciones patrimoniales (Venero Oblitas \& Samaniego Mollinedo, 2010).

- Objetivos específicos de la intervención

- La recuperación estructural y funcional de los diversos componentes de las plataformas

- Restituir el esquema de uso espacial original, a través de la liberación de los diferentes agregados que alteran la integridad del monumento

- Detener las causas de deterioro y minimizar su acción sobre la estructura del monumento, a fin de garantizar su estabilidad

- Establecer accesos y vías de circulación peatonal, proporcionando alternativas que no agredan al patrimonio y que faciliten los enfoques sociales del proyecto

- Recuperar el sistema de riego matriz y habilitar los canales secundarios que se encuentren en buen estado de conservación

- Implementar un sistema de riego tecnificado que minimice los impactos sobre los paramentos de las plataformas

\section{Factores de deterioro identificados}

La ganadería poscolonial, que fue insertada para acelerar los procesos agrícolas que tienen lugar en estas plataformas, minimizó el uso de herramientas artesanales de parte de los labradores, y generó actividades que desfiguraron las mismas. Así, para facilitar el acceso, se abrieron caminos por medio de muros y paramentos de factura inca, y se deshabilitaron sectores de producción para habilitar tramos de paso, lo que produjo la pérdida total de las plataformas.

La agricultura que se desarrolla en este sector era una de las principales fuentes de ingresos económicos para los pobladores locales. Sin embargo, su mal manejo generó impactos irreversibles en las estructuras y en el paisaje de este sector. Así, el nivel de destrucción en el que se encontraron los diferentes componentes del mismo se debe a la explotación agrícola desmedida y el empleo de maquinaria con estos fines.

La sostenibilidad de los sistemas agropecuarios y la afección directa del medio ambiente en estas tierras, generalmente más productivas, se torna más complicada ante el actual crecimiento de la población y la presión que esto ejerce sobre el uso de la tierra. Este es uno de los principales factores de deterioro del sistema de andenerías.

Además, se clasifican algunos otros factores de deterioro:

- Factores antrópicos: debido a la acción del hombre, se identificó la pérdida de cabeceras de muro, así como la ruptura de estos para la apertura de accesos para el tránsito de vehículos y animales, y cosecha de productos.

Construcción de viviendas precarias y cercos adosados a muros de andenes. 


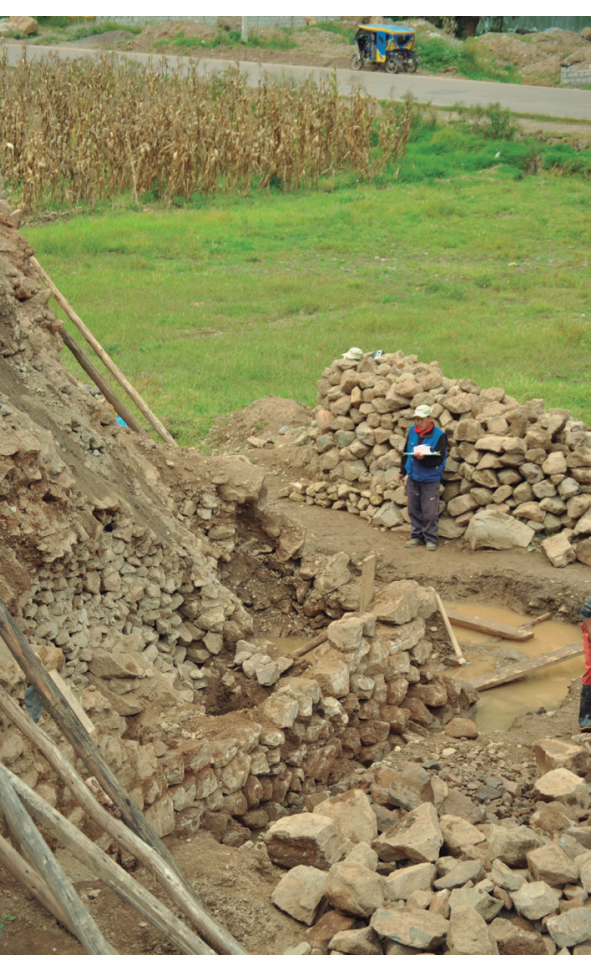

Figura 17. Vista de sectores de andén con problemas de estabilidad Fuente: Archivo fotográfico Shelby Paz, 2010.
Las plataformas agrícolas no eran aprovechadas al 100\%. Tras la pérdida del sistema de canales y soluciones hidráulicas originarias eran abandonadas.

El riego por inundación fue una técnica empleada en estas plataformas, que trajo consigo fallas estructurales considerables por el exceso y cambio de carga sobre los andenes. Cambiar este esquema estructural estático y permeable trajo como consecuencia la pérdida de cohesión y resistencia de los materiales de junta (Paz Valencia \& Samaniego Mollinedo, 2011) (ver Figura 17).

- Factores extrínsecos: en gran parte del monumento se observó abundante presencia de litolíquenes ferrosos y musgos presentes de manera invasiva sobre la superficie del paramento, los cuales, a su vez, forman parte de la pátina del tiempo en estas estructuras.

La presencia de agua subterránea debilitó la capacidad estructural del suelo. Asimismo, la humedad generada por aguas estancadas en los canales de riego ocasionó la putrefacción de algunos elementos estructurales, cuya consecuencia fue el colapso de algunas secciones de muros.

- Factores intrínsecos: la exfoliación de los elementos líticos, debido a los procesos de cambio de temperatura, y la excesiva humedad detectadas en este sector, produjeron fracturas y grietas en los elementos líticos.

El alto contenido de sales en la estructura cristalográfica del material lítico permite observar los procesos de meteorización en las piezas hechas de este material.

Durante las observaciones y estudios realizados para la elaboración del expediente técnico, se destacó la falta de un manejo adecuado y el cuidado de las prácticas de riego empleadas sobre las plataformas (por inundación ${ }^{2}$ ) con fines agrícolas. Esta práctica desmesurada fue la causa principal de las patologías estructurales identificadas, así como de los colapsos y pérdidas de paramentos.

\section{Procesos de intervención}

El proceso de intervención se ejecutó aproximadamente durante tres años. Pese a que aún no ha concluido la intervención en su totalidad, se tiene una lectura clara de los objetivos de la misma; se ha logrado habilitar en uso el 70\% del área planteada en el proyecto. Durante el primer año, la intervención se implementó de forma complementaria a los componentes sociales y ambientales planteados en el expediente técnico. Durante esta etapa, el desarrollo de actividades en obra se destaca de la siguiente forma (Venero Oblitas \& Samaniego Mollinedo, 2010):

- Implementación de salvaguardas sociales y ambientales, un aporte importante para el fortalecimiento de la identidad y la revaloración de los sistemas endógenos de la comunidad en relación a su patrimonio.

- Componente arqueológico. Aportó datos importantes al proyecto mediante la investigación y exploración del sitio. La intervención previa, mediante el proyecto de investigación, logró establecer parámetros, y orientar las intervenciones restaurativas en base a evidencias y hallazgos, como estructuras soterradas, que enriquecieron la puesta en valor del sitio.

Los trabajos de exploración arqueológica lograron evidenciar la tipología constructiva de las plataformas. Estas se encuentran constituidas por una estratigrafía en base a grandes bloques de piedra, sobre los cuales se diseñaron diversos estratos de relleno

2. En esta modalidad tradicional, una vez que la parcela se ha llenado de agua, se cierra la entrada a la misma, por lo que el agua no circula sobre el suelo, sino que se infiltra o evapora. Este tipo de riego, además de consumir mucha agua, tiene también un efecto poco deseable de compactación del suelo. 
artificial, hasta llegar a un suelo de tierra preparada para el cultivo. Estos, en su conjunto, formaron taludes que alcanzaban diversas alturas con estratigrafías diferenciadas. Se pueden desatacar algunas de las características acerca de la construcción de estos andenes, que permitieron observar la constitución característica de los paramentos de andén, así como la conformación estructural de estos.

En la base del Anden B, se logró realizar trabajos estructurales de refuerzo, como encuentros de esquinas, mediante un ingenioso sistema de arriostre elevado triangular, cuya cara posterior respondía a un semiarco. Cada estructura fue trabajada de manera singular, por lo cual exigió, en muchos de los casos, intervenciones específicas y de singular tratamiento (ver Figuras 18, 19 y 20 ).

- Componente arquitectónico. Se llevaron a cabo las intervenciones de restauración y conservación de los andenes bajo los objetivos planteados, mediante la compilación de los diversos aportes y aproximaciones de los diferentes profesionales y técnicos. La interpretación arqueológica de los elementos presentes en el sitio permitió tener una lectura clara de la función de cada uno de ellos.

- Estudios complementarios. Debido a la presencia de agentes biológicos y la excesiva presencia de humedad en los paramentos, se desarrolló un estudio de petrografía y litolíquenes de los elementos líticos del muro de los andenes. Dicha evaluación del material lítico, mediante estudios biológicos, permitió definir la tipología de litolíquenes y musgos presentes en los referidos muros.

Parte de los principios aplicados durante los procesos de restauración fueron el empleo de materiales de asentado y piezas líticas compatibles. Así, se obtuvo un mortero mejorado mediante el uso de hawank'ollay, que eleva sus niveles de cohesión, ductilidad e impermeabilidad. Este finalmente fue empleado como mortero de junta y asentado de piedras de muro (Venero Oblitas \& Samaniego Mollinedo, 2010).

La intervención de los paramentos siguió rigurosamente la metodología planteada a detalle en el proyecto:

- Se delimitaron las zonas de intervención conforme a la programación y planificación de la propuesta de trabajo para el periodo de ejecución durante el primer año.

- Esta delimitación permitió realizar una identificación de patologías sustentada en el expediente técnico. Luego de una limpieza manual de cada tramo identificado, se logró encontrar y agregar algunas patologías que no fueron consideradas al inicio, debido a la excesiva presencia de maleza y arbustos en la zona.

- La identificación de las principales patologías en los paramentos permitió elaborar un registro fotográfico previo a la intervención. Así, se pudo tener un registro de las intervenciones de forma progresiva, que facilitó el desarrollo de los principales trabajos en el sector, como la consolidación de muros de piedra, la recomposición de muros y canales verticales, la restitución de muros con material propio como material nuevo, y la recomposición y restitución de escaleras de material lítico.

- Se identificaron los principales componentes arquitectónicos del sector de forma integral. Así se identificó, en la base de este sistema de andenerías, la presencia de un singular sistema de circulación apoyado en una vereda empedrada, así como un juego sinuoso de peldaños embutidos en muros, canales verticales y sarunas.

Durante la ejecución de trabajos sobre los paramentos de contención a nivel general se definieron los siguientes detalles:

- Toda intervención de restitución realizada con piedra propia (producto de la anastilosis), se realizaría con una pestaña de 2" hacia el interior, desde la cara del muro original hacia atrás, lo cual facilita su lectura como un tramo restituido.



Figura 18. Recuperación de estas estructuras que a manera de zarpas se ubicaron al pie del andén B3. Aparentemente en la parte central la compactación es diferente, dejando un espacio para el tránsito Fuente: Archivo fotográfico Shelby Paz, 2010. 
devenir VOL. 3, N5, ENERO - JUNIO 2016, PP. 133-154 - EstudIOS I ISSN 2312-7570

UNIVERSIDAD NACIONAL DE INGENIERÍ, LIMA

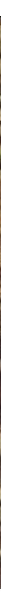



Figuras 19 y 20. Evidencias de ambas estructuras deterioradas a causa de la presión ejercitada por la tierra acumulada sobre ellas. Se realizaron trabajos de corte y eliminación de desmonte para luego atender su recomposición y restitución, previo reticulado y codificación

Fuente: Archivo fotográfico Shelby Paz, 2010.
- Los trabajos de restitución con material nuevo (sectores perdidos o colapsados) se realizaron dejando una pestaña de 3 " hacia el interior, desde la cara superior del paramento, además de la distinta tonalidad del material lítico.

- Para el caso de la restitución de piezas como sarunas, se decidió realizar una seña, que fue una marca de bisel de 4" en cada pieza restituida.

- La intervención se apoyó en diferentes estudios realizados durante el proceso de trabajo, como fue el análisis petrográfico del material de los andenes. Este arrojó una clasificación de material diorita de hornblenda, de fragmentación moderada y meteorizable, a causa de la acción erosiva del viento y la humedad. Como consecuencia de esto, muchas piezas no se encuentran fragmentadas por fuerzas de tracción sino que, por el contrario, se encuentran fragmentadas hacia las caras frontales del paramento. Esta identificación facilitó la ubicación de canteras con el mismo tipo de piedra.

- El proceso de intervención se inició de manera ascendente. Los primeros trabajos realizados en estos paramentos tras las exploraciones arqueológicas permitieron observar la existencia de cimentación no mayor a 1.20 metros, razón por la cual se perdió la estabilidad de gran parte de los paramentos iniciales.

- Se implementaron sendas para la circulación peatonal. Mediante una capa de cascajo se logró diferenciar el área agrícola de cada plataforma.

Es importante destacar que todos los procesos de restauración contaron, adicionalmente, con un trabajo de monitoreo arqueológico de forma permanente durante las obras, que se complementó con el componente de investigación arqueológica. Así, se facilitó la obtención de material y evidencia en los andenes, lo que permitió llevar a cabo las intervenciones arquitectónicas (ver Figura 21).

En los andenes se observó la presencia de abundante maleza, arbustos, árboles y raíces antiguas, elementos que degradan el núcleo de la estructura de soporte y los elementos líticos que lo conforman. Así, las obras de intervención se iniciaron con la eliminación de esta maleza, una limpieza general y eliminación de basura dentro de los andenes, así como la eliminación de raíces y la limpieza de las zonas de excavaciones. Una vez concluido el proceso de excavación arqueológica, se dio paso a las obras, y acciones de recuperación y restauración arquitectónica (ver Figura 22).

La intervención en los paramentos de los andenes consistió en la recomposición, la restauración y, en menor medida, la consolidación de dichas estructuras. Durante estos trabajos, se conservaron las características de la mampostería y los elementos líticos constitutivos, así como la tecnología estructural. La principal característica de esta 

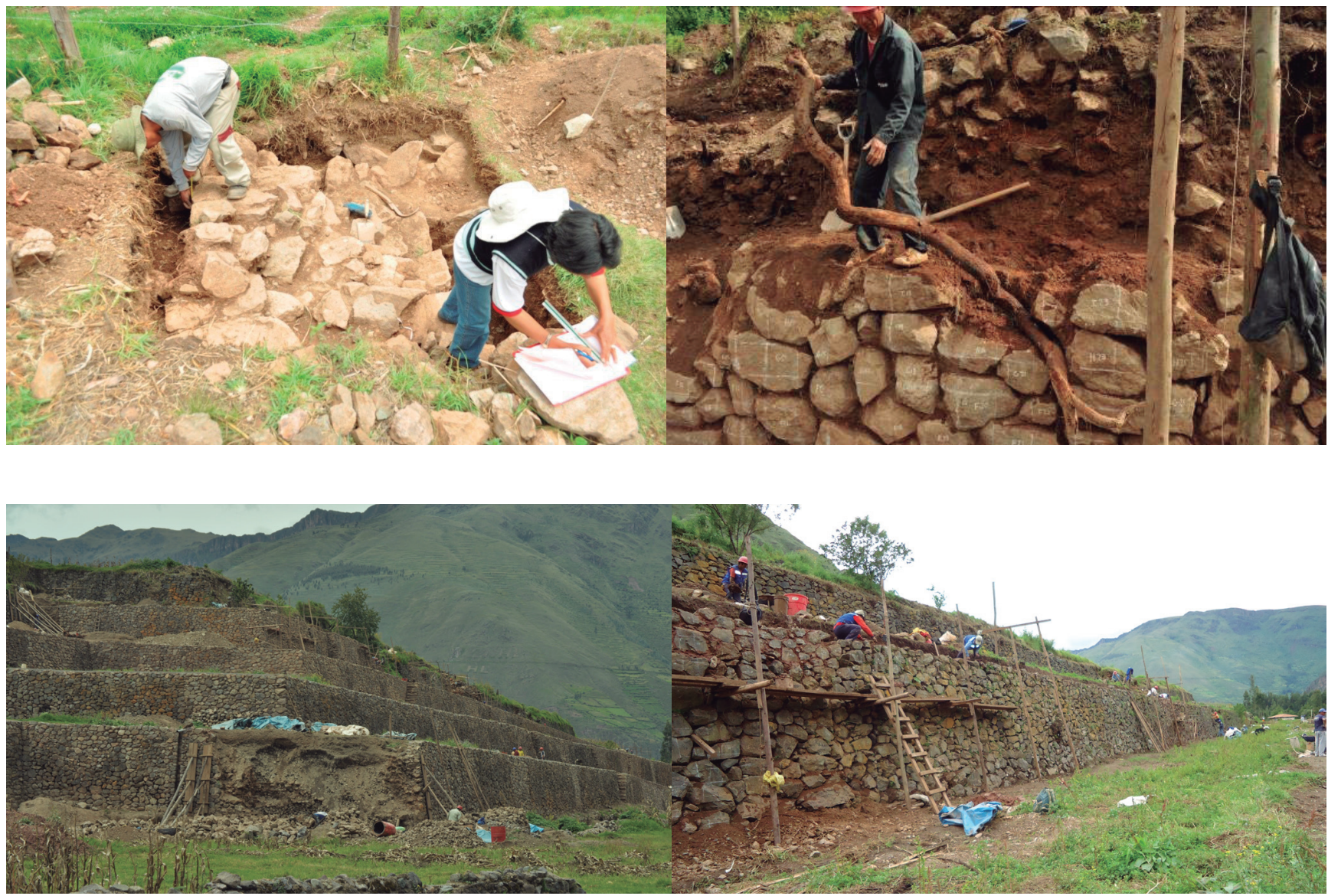

mampostería es la inclusión de algunos elementos de amarre y soporte estructural (Paz Valencia \& Samaniego Mollinedo, 2011).

Durante la intervención no se registraron deterioros, como el asentamiento o hundimiento, de cimiento o muros. Esto se reveló en la profundidad de los cimientos hallados, con un promedio de cimentación de 1.80 metros de altura. Estos cimientos fueron trabajados con piezas pétreas de regular tamaño, que guardan correspondencia con la lógica de emplazamiento y el perfeccionamiento en la construcción de muros de contención.

Los trabajos realizados en el primer andén (B) contemplaron la restitución de los paramentos con material propio, con lo que se recuperó el nivel perdido de esta plataforma, la cual asegura su estabilidad y recobra el sistema de respiración de estos muros mediante la incorporación de cangrejeras a un nivel de 0.80 metros de altura. Muchos de estos elementos fueron retirados de su emplazamiento original para ser empleados en otros sectores.

Se logró evidenciar un sistema de escalinatas embutidas, en las cuales se realizaron trabajos de recomposición y restitución de algunos pasos, con lo que se pudo habilitar la circulación a través de estos.

Luego de la exploración arqueológica, se evidencia en la primera esquina de los andenes una estructura singular de amarre. Esta estructura no posee el relleno tradicional $y$, por el contrario, hacia la parte posterior presenta una especie de arriostres que lo sostienen. Eran tres arriostres de 1.20 metros de altura, que dejaban entre sí espacios con relleno suelto sin mortero, los cuales drenan las aguas subterráneas y ojos de agua ubicados entre ellos, y a un nivel inferior de los cimientos (ver Figura 23).
Figura 21. Unidades de excavación arqueológica

Fuente: Archivo fotográfico Shelby Paz, 2010.

Figura 22. Retiro de árboles incrustados en el paramento

Fuente: Archivo fotográfico Shelby Paz, 2010.

Figura 23. Trabajos de reestructuración de la esquina del muro $B$

Fuente: Archivo fotográfico Shelby Paz, 2010.

Figura 24. Estado de deterioro del paramento $B$, restituido en casi el $40 \%$ de su longitud. Se rescató así la morfología del conjunto, y la lectura de las plataformas.

Fuente: Archivo fotográfico Shelby Paz, 2011. 
devenir VOL. 3, N5, ENERO - JUNIO 2016, PP. 133-154 - EstudIOS I ISSN 2312-7570

UNIVERSIDAD NACIONAL DE INGENIERÍ, LIMA

Los sectores identificados con mayor deterioro fueron los paramentos alrededor de los canales verticales, los cuales perdieron gran porcentaje del mortero de junta, lo que desestabilizó su estructura. Mediante procesos de anastilosis estos paramentos fueron recompuestos.

De forma general, dentro del proceso de intervención de los andenes, se realizaron trabajos de consolidación, recomposición y restitución de muros, mediante la utilización de mortero de arcilla mejorada.

Hacia el oeste, los tramos de andenes con mayor deterioro y pérdida fueron aquellos en los cuales se realizaron aperturas de caminos y tramos de acceso vehicular, que les hizo perder su continuidad y nivel. Para la recuperación de estos, se realizaron trabajos limpieza, remoción de desmonte y corte de terreno dentro de las plataformas, lo que permitió observar que el nivel de pérdida de los andenes era de un 30\% (ver Figuras $24,25,26,27,28,29,3031,32,33,34,35,36$ y 37 ).


Figura 25. Esquina del muro B, estructuras en forma de peine, sistema de refuerzo a filtraciones subterráneas existentes bajo estas estructuras Fuente: Archivo fotográfico Shelby Paz, 2011.

Figura 26. Restitución de muros con material nuevo, secciones colapsadas Fuente: Archivo fotográfico Shelby Paz, 2011.

Figura 27. Recomposición de muro de anden y nivelación de terreno Fuente: Archivo fotográfico Shelby Paz, 2010.

Figura 28. Identificación de problemas estructurales en paramento, colapsos y pérdida de material original del mismo

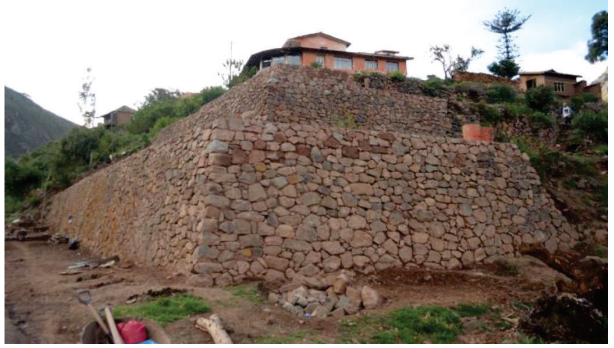

Fuente: Archivo fotográfico Shelby Paz, 2010.

Figura 29. Escalinata 02, Anden B3. Proceso de recuperación de escalinatas de piedra Fuente: Archivo fotográfico Shelby Paz, 2010.

Figura 30. Vista de escalinata de piedra luego de la limpieza preliminar de malezas Fuente: Archivo fotográfico Shelby Paz, 2010.

Figura 31. Recomposición y consolidación de esquinas, evidencias que pese al tiempo aún conservan su monumentalidad y composición estructural

Fuente: Archivo fotográfico Shelby Paz, 2010. 
Parte de los trabajos que demandaron mayor mano de obra y un incremento del presupuesto inicial, planteado en el expediente para estos trabajos, fue la partida de recomposición de muros, los cuales fueron identificados de forma veraz como resultado de las exploraciones arqueológicas.

Es importante indicar que la cantidad de tramos faltantes en la continuidad de los muros de andén, fueron mayores a los indicados en el expediente técnico. Esto generó una serie de imprevistos y demandó mayores metrados.

En la parte superior de los andenes, luego de concluir la intervención del paramento, como parte final de la misma, se realizó un trabajo de emboquillado de las cabeceras de muro, con una mezcla de arcilla, tierra cernida, gravilla (5/8) y cemento. Este proceso formó parte de la protección de las cabeceras de muro contra las filtraciones, las cuales posteriormente fueron parte de las áreas de tratamiento para la circulación y el tránsito peatonal, incluidas en la propuesta de circulación y visita turística (ver Figuras 38, 39, 40 y 41).

\section{Implementación de salvaguardas ambientales y sociales}

La elaboración del Proyecto Vilcanota requirió prestar atención a temas ambientales y sociales, los cuales se orientarían a que los proyectos de inversión impulsaran enfoques de desarrollo sostenible. La ejecución de trabajos complementarios de manera multidisciplinaria se desarrolló de forma tal que se integraron a los trabajos de restauración y puesta en valor durante el primer año. Así, se integraron transversalmente a los lineamientos establecidos por organismos internacionales que priorizan las practicas ecológicas y la calidad de vida de las comunidades, tales como ICOMOS o UNESCO.

\section{Salvaguardas sociales}

Esta actividad se implementó tomando como base el estudio previo realizado dentro del Proyecto de Facilitación Social, parte del Proyecto General. Se desarrolló un acercamiento a la situación social dentro de la comunidad de Urco y se establecieron las temáticas principales vinculadas a nuestra intervención en campo, que posteriormente serían temas de desarrollo para los talleres con la comunidad de Urco: Patrimonio e Historia - importancia; Uso y Manejo de los Andenes; Manejo de Cultivos; Sistema de Riego.

De forma paralela a la ejecución de la obra física, se desarrollaron talleres con el apoyo de un equipo multidisciplinario e interinstitucional, conformado por la Municipalidad Provincial de Calca, el Ministerio de Cultura y algunas instituciones dedicadas a la agricultura, organismos entre los cuales se buscó sinergia para optimizar los resultados.

Este componente debía lograr el acercamiento de la comunidad al patrimonio para generar su concientización y revalorización, con incidencia en el sistema de andenes y los otros trabajos que se habían estado realizando. Se activaron mecanismos de visita y guía en el sitio, y se capacitó a los integrantes de la comunidad para realizar dichas actividades.

Los logros más resaltantes de este componente se dieron con la aceptación de la demolición voluntaria de dos viviendas construidas, las cuales estaban adosadas a los muros de los andenes, así como con la recuperación del festival de culto al agua, denominado Unu Urco, que buscaba promover el uso responsable del agua y el sistema de andenerías. Así se potenciaría su oferta turística (ver Figuras 42 y 43 ).

\section{Salvaguardas ambientales}

Esta actividad se implementó sobre la base del desarrollo del Plan de Monitoreo Ambiental establecido en el Proyecto de Restauración y Puesta en Valor. Incluyó la capacitación de los pobladores en temas de reciclaje de desechos, se desarrollaron

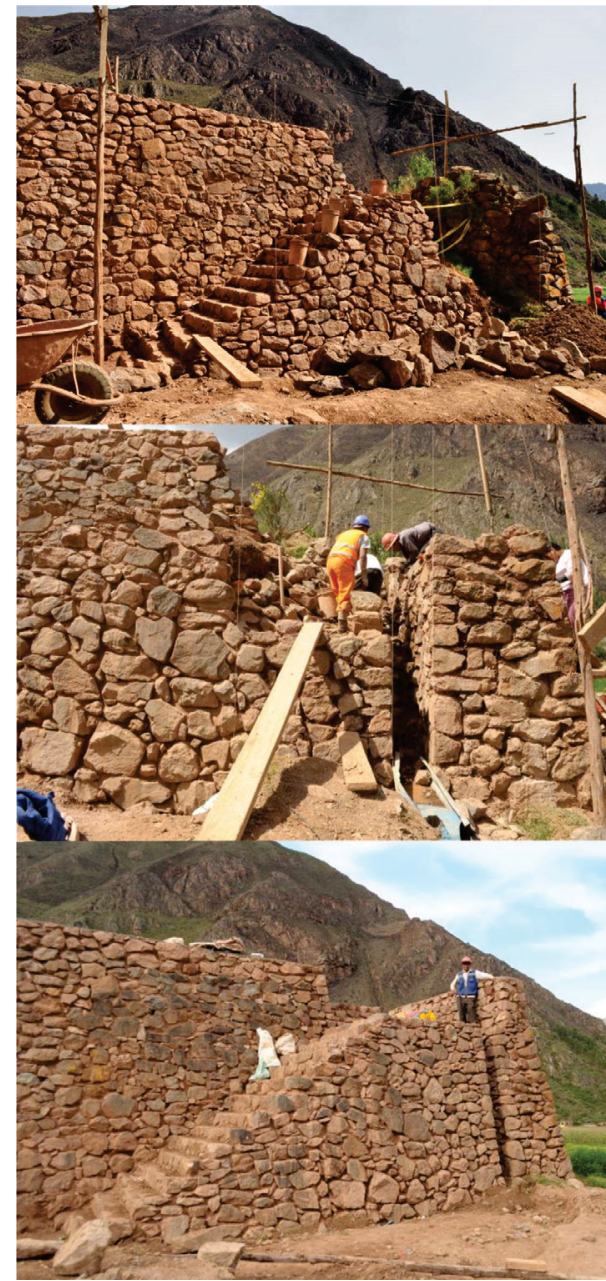

Figura 32. Anden C2. Proceso de excavación arqueológica, trabajos de limpieza y eliminación de maleza, que permitieron evidenciar las estructuras de escalinatas embutidas en este sector

Fuente: Archivo fotográfico Shelby Paz, 2010. 

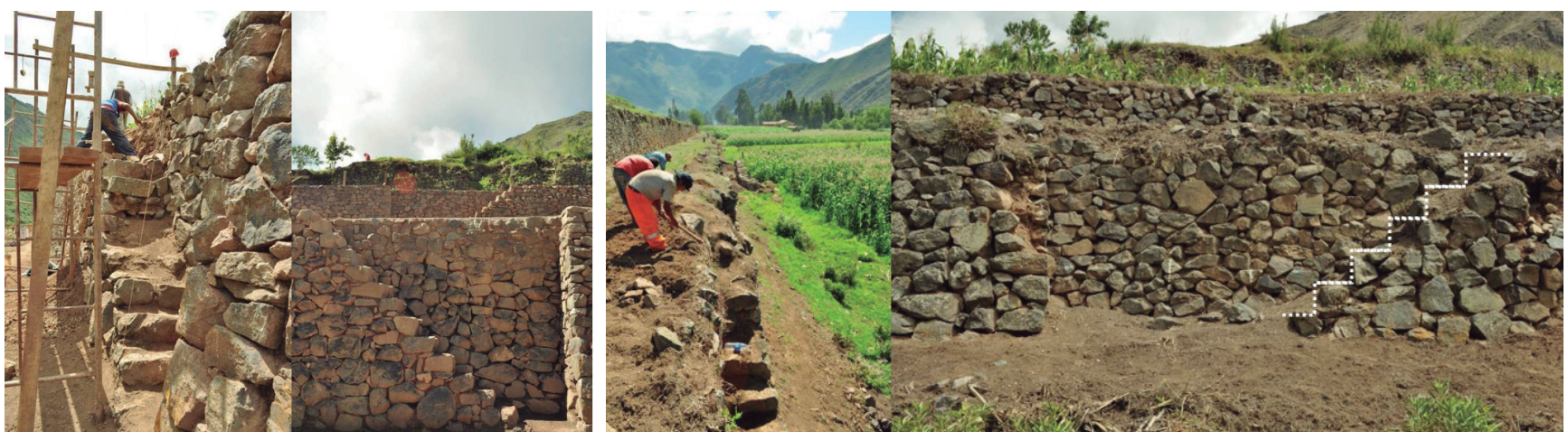

Figura 33. Trabajos de recomposición de escalinatas. Se observa el sistema de trabajo en base a guías empleadas mediante la colocación de balizas; cada pieza fue identificada y codificada.

Fuente: Archivo fotográfico Shelby Paz, 2010.

Figura 34. Estado de conservación en la que se encontraron los primeros pasos de las escalinatas, luego de las exploraciones arqueológicas, que probablemente por el uso agrícola de estas plataformas fue soterrado con el paso del tiempo

Fuente: Archivo fotográfico Shelby Paz, 2010.
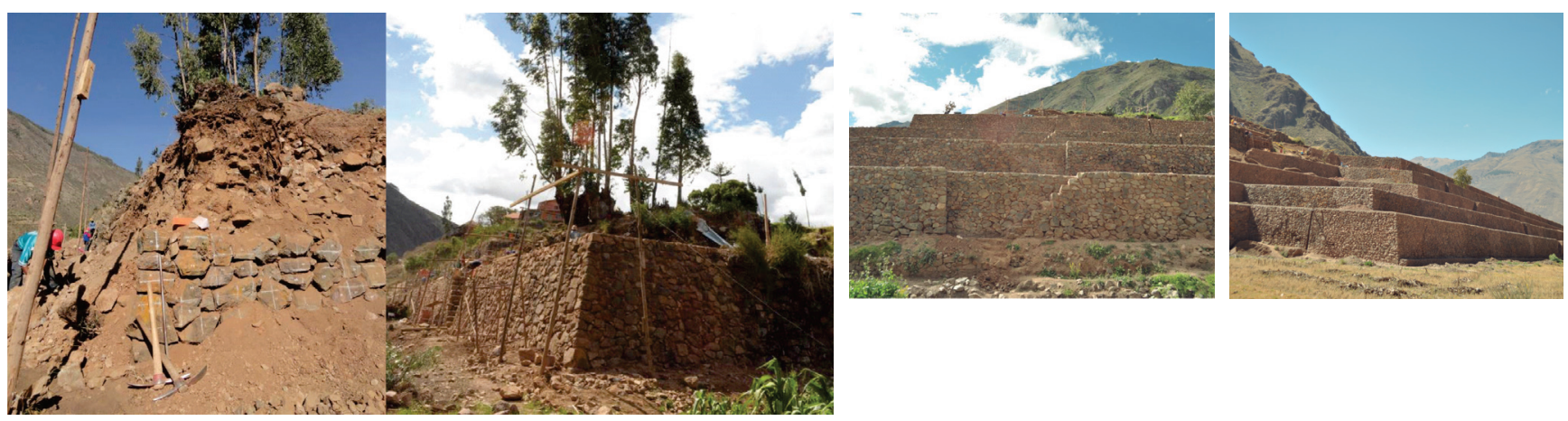

Figura 35. Restitución y reestructuración de esquinas de andén, colapsados por la falta de mantenimiento y la proliferación de maleza

Fuente: Archivo fotográfico Shelby Paz, 2010.

Figura36. Escaleras embutidas, luego de su restauración. La composición formal de las escalinatas es armoniosa, probablemente de uso ceremonial; se logró rescatar no solo su lectura estructural sino su composición arquitectónica.

Fuente: Archivo fotográfico Shelby Paz, 2010.

Figura 37. Anden B, C y D. Posterior a su restauración, plataformas recuperadas al $100 \%$, reforzamiento de las esquinas

Fuente: Archivo fotográfico Shelby Paz, 2011. campañas de limpieza de los sectores aledaños a los andenes, y se elaboró compost y productos derivados del reciclaje.

Un logro importante fue la participación de la comunidad en talleres de intercambio de experiencias con el Instituto Nacional de Innovación Agraria (INIA), los cuales transmitieron sus experiencias en implementación de sistemas alternos de regadío en vez de sistemas de goteo y cambio de cultivos.

Se propuso una herramienta importante para la rehabilitación de los andenes: un plan de manejo ambiental que permitió sopesar los impactos producidos por las alteraciones que se realicen al entorno paisajístico mediante el desarrollo de actividades de intervención y posintervención.

Se consideró los muestreos de las vertientes de ríos y afluentes para el sector de Urco, especialmente para los andenes, a través de la realización de estudios físico-químicos del agua. Asimismo, se tomó en consideración un seguimiento al manejo de agua, la calidad de suelos, el manejo de residuos sólidos y el impacto de la intervención ante la pérdida de la cobertura vegetal que existía anteriormente.

En los talleres con la comunidad se contó con el apoyo de instituciones como Plan Meris con el tema de manejo de cultivos; y el INIA, respecto del tema de sistema y 



manejo de riego en andenes. Esto generó un intercambio de conocimientos con los pobladores de la comunidad de Urco, los cuales visitaron la zona experimental y de prueba de nuevos cultivos.

Finalmente, se plantearon estrategias de conservación ambiental del sector intervenido con la implementación de selección de desechos mediante la colocación de basureros para material orgánico e inorgánico. Su tratamiento incluyó el color y los idiomas castellano y quechua; de esta manera, se generó la apropiación de este material por parte de la población (Venero Oblitas \& Samaniego Mollinedo, 2010) (ver Figuras 44, 45 y 46).

\section{Reflexiones}

- El sistema de riego por inundación ha significado un deterioro muy rápido en los paramentos de los andenes. Esta metodología de riego durante mucho años ha sido perjudicial para muchos de los sistemas de andenería que existen en el valle, por lo que es necesario intensificar las acciones que minimicen estas actividades y permitan un uso provechoso del recurso.

- El planteamiento fue una propuesta innovadora que trajo a discusión la puesta en uso social de una zona arqueológica con características agrícolas y comunidades involucradas; por ello, el Ministerio de Cultura tuvo algunos reparos en la aprobación de
Figura 38. Vista de los andenes luego de concluido el primer semestre de intervención durante el año 2010 Fuente: Archivo fotográfico Shelby Paz, 2010.

Figura 39. Vista de los andenes luego de concluido el primer semestre de intervención durante el año 2011 Fuente: Archivo fotográfico Shelby Paz, 2011.

Figura 40. Vista del sistema de andenería concluido el último semestre de intervención durante el año 2011 Fuente: Archivo fotográfico Shelby Paz, 2011. 
principal el mantenimiento y protección del sistema de andenerías, lo que exigiría a su comunidad mayor conocimiento respecto a diferentes aspectos vinculados al manejo, gestión y protección del sitio.

- Los esfuerzos vertidos para la recuperación de este sistema de andenerías, la cual además consideró su uso social, resultaron en un modelo para las intervenciones que vinculan a las comunidades ancestrales. Sin embargo, se debe resaltar que es necesario implementar un grupo de salvaguarda, manejo y control con los pobladores de Urco, e incentivar su participación a través de la revaloración del valor patrimonial del conjunto.

- Es importante el establecimiento de sinergia entre los gobiernos regionales, los gobiernos locales, y las instituciones vinculadas a la protección del patrimonio y promoción turística, a fin de buscar alternativas que logren una participación activa del gobierno local dentro del ámbito del proyecto; esto permitiría desarrollar un nivel de concientización multidisciplinario. Ello debe ocurrir a todo nivel, pues no solo se trata de una puesta en valor del monumento sino de un proyecto que ha de lograr revalorar la identidad de la comunidad.

- La inserción de los pobladores del sector al grupo humano de trabajo ha permitido, dentro de la comunidad, la apropiación de las actividades desarrolladas en el proyecto, así como la capacitación de los pobladores.

- Este proyecto buscó trascender en las prácticas tradicionales de cultivo al reforzarlas para mantener y orientar la agricultura andina de la mano con la protección de su patrimonio cultural; esto los orientó a una producción agroecológica y fomentó un producto orgánico con una base de promoción turística propia, que aprovecha la riqueza cultural de este sector y maneja diversas alternativas de regadío.

- El mantenimiento de una rica diversidad, los parámetros del sistema agroecológico relacionados con las prácticas agrícolas y los sistemas de producción se consideran de suma importancia para que las futuras oportunidades de un mercado orgánico, servicios ambientales y el encanto paisajístico del lugar sean una oportunidad económica para el ecoturismo; este también se reforzaría con el encanto arqueológico que se encuentra en los andenes de Urco.

- Es necesario señalar que el estado de deterioro en el que fue encontrado el monumento fue elevado. Algunas zonas prácticamente se perdieron en su totalidad. El material empleado en el rescate de este monumento se incrementó debido a la inexistencia de muchas zonas de este. La intervención contemplada dentro de los parámetros que rigen las intervenciones de restauración y puesta en valor de monumentos arqueológicos estuvo estrechamente ligada a las normas establecidas desde la Carta de Atenas y documentos de los cuales el Perú es suscritor activo.

- El patrimonio cultural procura incluir contextos de preservación en los procesos económicos que se dan en cada ciudad; la falta de planes de manejo y estrategias de manejo para estos bienes culturales se convierte en un problema inmanejable, ya que no permiten integrar un aprovechamiento turístico sostenible del bien cultural que considere además la preservación y protección de sus comunidades. Estas últimas se encuentran directamente vinculadas entre sí por medio de la revaloración de sus costumbres y el fortalecimiento de su identidad, y la recuperación del espacio para sus actividades no solo agrícolas sino ceremoniales, ya que actualmente lo usan para desarrollar la ceremonia del Unu Urco.

- En términos generales la intervención logró una revaloración del bien material, que finalmente ensalzó el valor inmaterial del monumento y le otorgó un valor de sitio; su puesta en valor potenciará los valores asentados de identidad y cultura, y le retornará el significado funcional de originalidad con el que fue concebido.



Figuras 44 y 45 . Talleres de capacitación y recojo de basura por la localidad

Fuente: Archivo fotográfico Ana Oviedo a, 2011.

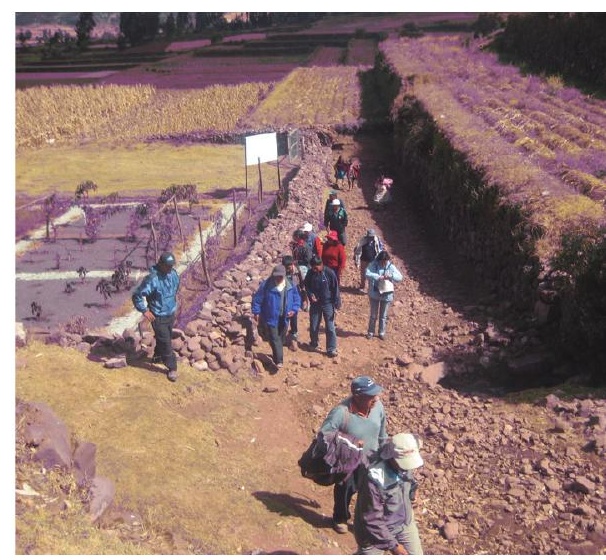

Figura 46. Visita de campo con los comuneros a los centros de prácticas de riegos alternativos del INIA

Fuente: Archivo fotográfico Ana Oviedo, 2011 
- La rehabilitación de infraestructura agrícola prehispánica debe compatibilizarse con los conceptos de una restauración apropiada y respetuosa, tanto a nivel tecnológico como constructivo. Sin embargo, uno de sus mayores retos es la inclusión de sus comunidades en la participación activa de la conservación de actividades agrícolas y el mantenimiento de las estructuras, de forma que los incluya participativamente en el ejercicio continuo y activo de prácticas tradicionales.

- Todos los elementos son revalorados y rescatados gracias a la intervención oportuna y eficaz, en un afán de rescate y puesta en valor de todos los valores agregados de este complejo; esto los pone en evidencia para los visitantes de este sistema de andenerías, y les da una lectura integral; ello gesta, de este modo, un nuevo enfoque y oportunidades de investigación del significado de estas estructuras, así como construcciones de nuevas hipótesis acerca de quiénes y por qué realizaron, en realidad, tan espléndidos trabajos.

\section{Referencias}

Betanzos, J. d. (1987). Suma y narración de los incas. (M. d. Rubio, Trad.) Madrid, España: Atlas.

Canziani, J. (2009). Ciudad y territorio en los Andes: Contribuciones a la historia del urbanismo prehispánico. Lima, Perú: Fondo Editorial, Pontificia Universidad Católica del Perú.

Dorado Alegre, J. d. (2010). Informe Final de Evaluación Geológica del Proyecto de Restauración y Puesta en Valor de la Zona Arqueologica Monumental Urco Sector I Qosqopata, PER Plan COPESCO - Cusco. Cusco: PER Plan COPESCO.

Gasparini, G., \& Margolies, L. (1997). Arquitectura Inca. (F. d. Urbanismo, Ed.) Venezuela: Universidad Central de Venezuela.

Herrera, A. (2011). La recuperación de tecnologías indígenas: Arqueología, tecnología y desarrollo en los Andes. Lima: Instituto de Estudios Peruanos.

Kendall, A. (1976). Descripción e inventario de las formas arquitectónicas inca. Revista del Museo Nacional, 42, 13-96.

Kendall, A., \& Rodríguez, A. (2009). Desarrollo y perspectivas de los sistemas de andenería en los Andes Centrales del Perú. Cusco, Perú: Centro de Estudios Regionales Andinos Bartolomé de Las Casas.

Instituto Nacional de Cultura. (2007). Documentos Fundamentales para el Patrimonio Cultural (I ed.). (M. d. Cultura, Ed.) Lima: Biblioteca Nacional del Perú.

Larios Villalta, C. R. (2009). Manual de criterios de Restauración para la arquitectura Prehispánica. Guatemala: Programa de Desarrollo de Peten para la Conservación de la Reserva de la Biosfera Maya. Guatemala: BID/PDCRBM.

Lumbreras, L. G., González, C. E., \& Águila, C. (2005). Arqueología y sociedad. Lima: Instituto de Estudios Peruanos.

Paz Valencia, S., \& Samaniego Mollinedo, J. (2011). Informe final del Proyecto de Restauración y Puesta en Valor de la Zona Arqueologica Monumental Urco Sector I Qosqopata, Calca. PER Plan COPESCO - Cusco. Años 2010-2011. Cusco: PER Plan COPESCO.

Petzet, M. (2000). Informe Mundial 2000 De Icomos Sobre Monumentos Y Sitios En Peligro. Recuperado el 26 de abril de 2015, de H@R! : Heritage at Risk / Patrimonio en Peligro: http://www. icomos.org/risk/world_report/2000/intro_spa.htm

Samaniego Mollinedo, J. (2012). Informe Final de Proyecto de Investigación Arqueologica en la Zona de Urco, Qosqopata, Calca. Cusco: PER Plan COPESCO.

Valcárcel, L., \& Rénique, C. (2013). Luis E. Valcárcel: del indigenismo cusqueño a la antropología peruana. Lima: IEP.

Venero Oblitas, D. \& Samaniego Mollinedo, J. (2010). Proyecto de Restauración y Puesta en Valor de la Zona Arqueologica Monumental Urco Sector I Qosqopata, Calca. PER Plan COPESCO. Cusco, Perú: PER Plan COPESCO

Victor, A. V. (1988). Historia del Cusco incaico, Tomo Il. Cusco, Perú. 